\title{
Evaluation of Anti - Anxiety, Sedative and Motor Co-Ordination Properties of Ganaxolone in Comparison with Diazepam in Rodent Models
}

\author{
B. L. Kudagi ${ }^{1}$, R. Pravin Kumar $^{2}$, S.K. Subani Basha ${ }^{3}$ \\ ${ }^{1}$ (Professor \& H.O.D., Department of Pharmacology, Narayana Medical College, Nellore, India) \\ ${ }^{2}$ (M.D. P.G. Student, Department of Pharmacology, Narayana Medical College, Nellore, India) \\ ${ }^{3}$ (M.Sc. P.G. Student Department of Pharmacology, Narayana Medical College, Nellore)
}

\begin{abstract}
Presently, treatment of anxiety with standard anti-anxiety drugs is associated with a number of shortcomings inviting us to study newer agents that would overcome these problems or search for the drugs or substances, which would enhance the efficacy or reduce the dose or toxicity of this standard anti-anxiety drug diazepam. Ganaxolone $(G N X)$ a positive allosteric modulator of $G A B A_{A}$ receptors, may represent a new treatment approach for anxiety. The present study was undertaken to evaluate the anti - anxiety effect of ganaxolone in comparison with diazepam and also to study their effect on sleep and motor co-ordination using elevated plus maze, thiopentone sodium induced sleeping and rotarod model in rodents. Wistar strain albino rats weighing 200-250 gm were used. Ganaxolone produced significant anti-anxiety effect compared to control group but the activity was less when compared to diazepam. The sedative and muscle relaxant properties of ganaxolone were weak as compared to diazepam. Ganaxolone had significant synergistic effect with diazepam by potentiating its actions, thereby providing a lead for exploring potential benefit of adding ganaxolone to decrease the dose of diazepam in the treatment of anxiety.
\end{abstract}

Keywords: Anxiolytic, diazepam, elevated plus maze, ganaxolone, rotarod

\section{Introduction}

Anxiety is a psychological and physiological state characterized by somatic, emotional, cognitive, and behavioral components, associated with significant disability (including educational and occupational) which has a negative impact on the quality of life [1]. Anxiety disorder is increasingly recognized as a highly prevalent and chronic disorder with onset during the teenage years, with an incidence of $18.1 \%$ and a lifetime prevalence of 28.8\%. [2] Individuals are spending lot of money to rid themselves of anxiety. The cost of visits to physicians and utilization of health care in general by individuals with anxiety disorders, are double compared to those without anxiety disorders, even if the later is physically ill[3]. Anxiety has become a significant area of psychopharmacological research during this decade, as it affects around one-eighth of the total population of the world.

The three neurotransmitters primarily implicated in anxiety are GABA, serotonin and noradrenaline [4]. Evidence of GABAergic involvement in modulating anxiety is that certain classes of drugs such as the benzodiazepines, barbiturates and alcohol all bind to GABA receptors to increase its post-synaptic inhibitory effect and reduce anxiety. Benzodiazepines bind allosterically to the GABA receptor and have their own binding site. Additionally, benzodiazepine inverse agonists such as flumazenil decrease effects of GABA and cause anxiety [5]. Benzodiazepines are the prime class of compounds used in anxiety and they have remained the most commonly prescribed treatment for anxiety, but they can cause deterioration of cognitive functioning, sedation, muscle relaxation, ataxia, psychomotor impairment, confusion, anterograde amnesia, physical dependence and tolerance [6,7]. For these reasons, newer anti-anxiety drugs with better efficacy and decreased side effect profile is needed.

Therefore, pharmacological manipulations leading to increased levels of GABA (by inhibition of GABA degradation or reuptake) and/or positive allosteric modulation of the GABA receptor complex could be

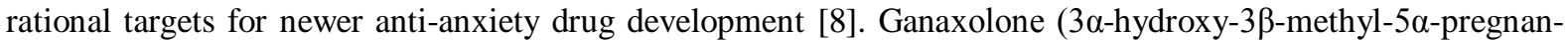
20 -one), the $3 \beta$-methyl analog of the progesterone metabolite allopregnanolone, is the first synthetic neurosteroid related agent to be evaluated in man for the treatment of epilepsy, which is now undergoing phaseIII clinical trials, acts as a powerful positive allosteric modulator of $\mathrm{GABA}_{\mathrm{A}}$ receptors, resulting in enhanced neural inhibition $[9,10]$. Hence, the present study was undertaken to evaluate the anti - anxiety effect of ganaxolone in comparison with diazepam and also to study their effect on sleep and motor co-ordination. 
1.1. Animals

II. Materials and Methods

Albino wistar strain rats weighing between 200-250gms were used, for this study. The animals were obtained from central animal house of Narayana Medical College, Nellore. They were housed in standard polypropylene cages. Animals were quarantined for 7 days before use and were excluded if the weight of rats were below $200 \mathrm{gms}$ and if they had any visible diseases. The animals were maintained under standard laboratory conditions $\left(12: 12 \mathrm{hr}\right.$ light: dark cycles and temperature $27^{\circ} \mathrm{C} \pm 1^{\circ} \mathrm{C}$ with free access to food and water ad libitum. All the experiments were carried out around the same time each day. All the experimental procedures and protocols were reviewed and approved by the Institutional Animal Ethics Committee (IAEC) of the institute, with protocol number 12/2010/NMC.

\subsection{Chemicals \& drugs}

All standard chemicals used in this study were of analytical grade. Pure form of ganaxolone \& Hydoxypropyl- $\beta$-cyclodextrin was obtained from Sigma chemicals. Stock solutions of ganaxolone for injection were made in $40 \%$ hydroxypropyl- $\beta$-cyclodextrin in water. By itself, $\beta$-cyclodextrin at concentrations as high as $50 \%$ failed to produce any anti-anxiety effect.

\subsection{Grouping}

To study the anti-anxiety activity of ganaxolone, elevated plus maze model was used and to study the effect on sedation and motor co-ordination thiopentone sodium induced sleeping time and rota rod apparatus were used respectively.

Groups are divided as follows,

Group I (Control group) was treated with $40 \%$ (w/v) hydoxypropyl- $\beta$-cyclodextrin $4 \mathrm{ml} / \mathrm{kg}$ body weight.

Group II received ganaxolone at a dose of $5 \mathrm{mg} / \mathrm{kg}$ body weight.

Group III received ganaxolone at a dose of $10 \mathrm{mg} / \mathrm{kg}$ body weight.

Group IV received standard drug, diazepam $2 \mathrm{mg} / \mathrm{kg}$ body weight.

Group V received ganaxolone at $5 \mathrm{mg} / \mathrm{kg}$ and diazepam $1 \mathrm{mg} / \mathrm{kg}$ body weight.

Separate groups of animals are used for different models and all the groups received the drugs intraperitonially, given $30 \mathrm{~min}$ prior to the start of the experiment. Concentration of drugs was so adjusted that all the animals in the group received the same volume of preparation throughout the study.

\subsection{Elevated plus maze (EPM) model for anxiety [11]}

The elevated plus maze apparatus consists of two opposite open arms (50x10) crossed with two closed arms of same dimensions with $40 \mathrm{~cm}$ high walls and these arms extend from a common central square $(10 \times 10$ $\mathrm{cm})$. The grip on the open arms is facilitated by a small raised edge $(0.5 \mathrm{~cm})$ around their perimeter to keep the rats from falling down. The entire maze is elevated to a height of $40 \mathrm{~cm}$ above the floor level. Rats were placed at the centre of the maze, $30 \mathrm{~min}$ after ganaxolone \& diazepam administration, facing one of the closed arms. Arm entries were counted when the animal placed all of its four paws on it. The procedure was conducted in a sound attenuated room. After each test, EPM was cleaned with spirit. During 5 min test period the following measures are taken,

1. The number of entries into the open arm.

2. The number of entries into the closed arm.

3. Time spent in the open arm.

4. Time spent in the closed arm.

\subsection{Thiopentone sodium induced sleeping time [11]}

The method employed in this study was described in Vogel. Thiopentone sodium 40mg/kg body weight was given to induce sleep; it was injected intraperitoneally to all groups after $30 \mathrm{~min}$ of administration of test and standard drug. The time interval between loss and recovery of righting reflex was measured as sleeping time, which was used as an index of hypnotic effect. The time interval between injection of thiopentone sodium and start of sleep was recorded as latency time.

\subsection{Rotarod test for motor co-ordination [11,12]}

Ganaxolone and diazepam were evaluated for motor co-ordination in an accelerating rotarod test. Animals were trained on the rotarod for one week (15minutes daily). Rats were acclimatized to the rotarod 30 min before the start of the experiment. Five groups of Rats were taken for the study (each group contains 6 Rats). Animals that remained on the rod for more than 5 minutes were selected for the study. The animals were then evaluated for motor coordination at an interval of 30 and 60 minutes, after 30 minutes of administration of test and standard drug. The time when each animal falls off from the rod for the first time and the number of 
falls were noted during the $5 \mathrm{~min}$ test period and was considered toxic if it fell from the rotarod three or more times.

\subsection{Statistical analysis}

The data was collected in case record forms and then entered into excel spreadsheet 2007. Statistical analysis was performed using Microsoft Excel - 2007 and Sigma Graph pad prism version-5 USA. Data was described as Mean \pm Standard deviation. One way ANOVA followed by Tukey-Kramer multiple comparison test was used for analysis of data between the five groups. For all inferential statistical tests a two tailed $\mathrm{P}$ value of $<0.05$ was considered significant.

\subsection{Anxiolytic activity}

\section{Results}

In elevated plus maze apparatus, diazepam treated rats showed significant increase $(\mathrm{P}<0.001)$ in the number of open arm entries, time spent in open arms and reduction in the time spent in closed arm. In ganaxolone $5 \mathrm{mg}$ and $10 \mathrm{mg}$ treated groups the percentage time spent in open arm was $34.77 \%$ and $43.50 \%$ respectively, which was less compared to diazepam treated group $(60.39 \%)$. Both ganaxolone $5 \mathrm{mg} / \mathrm{kg}$ and $10 \mathrm{mg} / \mathrm{kg}$ produced significant anti anxiety effect when compared to control group but the effect of ganaxolone $10 \mathrm{mg} / \mathrm{kg}$ was highly significant $(\mathrm{p}<0.001)$. In ganaxolone and diazepam combined group the percentage time spent in open arm was $46.83 \%$, signifying that time of stay in the open arm was more than ganaxolone $5 \& 10$ $\mathrm{mg} / \mathrm{kg}$ groups but not as much of diazepam treated group. Also, the mean number of entries to open arm was highly significant $(\mathrm{P}<0.001)$ with ganaxolone $10 \mathrm{mg} / \mathrm{kg}$ when compared to control group but less when compared with diazepam. [Table 1]

\subsection{Sedative property}

In thiopentone sodium induced sleeping, the latency period for control group was 9.5 minutes, for ganaxolone $5 \mathrm{mg}$ and $10 \mathrm{mg}$ it was 8.1 and 6.8 minutes, 2.8 minutes with diazepam and 4.1 minutes in ganaxolone and diazepam treated group. The difference between 5 and $10 \mathrm{mg} / \mathrm{kg}$ doses of ganaxolone was significant implying that higher dose of ganaxolone has a significant CNS depressant action. The mean sleeping time and latency period of ganaxolone $10 \mathrm{mg} / \mathrm{kg}$ was highly significant $(\mathrm{p}<0.001)$ compared to control but less compared to diazepam, implying sedative property of ganaxolone was not as efficacious as that of diazepam. [Table 2]

\subsection{Effect on motor co-ordination}

In rotarod test, ganaxolone $10 \mathrm{mg} / \mathrm{kg}$ significantly reduced the time spent by the animals on revolving rod when tested 30 minutes after drug administration as compared to control but there was no significance at 60 minutes. Ganaxolone $5 \mathrm{mg} / \mathrm{kg}$ did not show any significant effect at both 30 and 60 minutes. The motor incoordination effect of diazepam was highly significant as compared to control and ganaxolone groups. Ganaxolone and diazepam combined group produced less motor incordination than diazepam. [Table3]

Table 1: Effect of ganaxolone on elevated plus maze apparatus

\begin{tabular}{|c|c|c|c|c|c|c|c|}
\hline \multirow{2}{*}{$\begin{array}{l}\text { S. } \\
\text { no }\end{array}$} & \multirow{2}{*}{$\begin{array}{l}\text { Group \& Dose } \\
\text { (mg/kg, i.p) }\end{array}$} & \multicolumn{2}{|c|}{ Mean no. of entries in } & \multicolumn{2}{|c|}{$\begin{array}{c}\text { Mean time spent in } \\
(\mathrm{sec})\end{array}$} & \multirow{2}{*}{$\begin{array}{l}\text { Percentage } \\
\text { of open } \\
\text { arm } \\
\text { entries }\end{array}$} & \multirow{2}{*}{$\begin{array}{l}\text { Percentage } \\
\text { of time } \\
\text { spent in } \\
\text { open arm }\end{array}$} \\
\hline & & Open arm & $\begin{array}{c}\text { Closed } \\
\text { arm }\end{array}$ & Open arm & Closed arm & & \\
\hline 1. & Control & $4.5 \pm 1.05$ & $15.67 \pm 1.75$ & $76.67 \pm 9.54$ & $223.33 \pm 9.54$ & $22.31 \%$ & $25.56 \%$ \\
\hline 2. & Ganaxolone - 5 & $6.33 \pm 0.82 *$ & $13.50 \pm 1.05$ & $92.83 \pm 4.62 *$ & $207.17 \pm 9.69$ & $30.94 \%$ & $34.77 \%$ \\
\hline 3. & Ganaxolone - 10 & $9.17 \pm 0.75 * * *$ & $8.17 \pm 1.72$ & $130.50 \pm 8.17 * * *$ & $169.50 \pm 8.17$ & $52.88 \%$ & $43.50 \%$ \\
\hline 4. & Diazepam -2 & $12.50 \pm 1.05 * * *$ & $8.50 \pm 1.05$ & $181.17 \pm 8.23 * * *$ & $118.83 \pm 8.23$ & $59.52 \%$ & $60.39 \%$ \\
\hline 5. & $\begin{array}{l}\text { Ganaxolone } 5+ \\
\text { Diazepam } 1\end{array}$ & $11.50 \pm 1.05 * * *$ & $9.67 \pm 1.03$ & $159.50 \pm 8.46 * * *$ & $140.50 \pm 8.46$ & $53.07 \%$ & $46.83 \%$ \\
\hline
\end{tabular}

Table 2: Effect of ganaxolone on thiopentone sodium induced sleeping time

\begin{tabular}{|c|l|c|c|}
\hline S.NO & \multicolumn{1}{|c|}{$\begin{array}{c}\text { Group \& Dose } \\
(\mathbf{m g} / \mathbf{k g} \text { bw })\end{array}$} & $\begin{array}{c}\text { Mean time of onset for loss of righting reflex } \\
\text { / latency period (Min) }\end{array}$ & Mean sleeping time (Min) \\
\hline 1. & Control & $9.5 \pm 1.23$ & $43 \pm 2.5$ \\
\hline 2. & Ganaxolone - 5 & $8.1 \pm 1.34$ & $50 \pm 3.1$ \\
\hline 3. & Ganaxolone - 10 & $6.8 \pm 1.47 * *$ & $67 \pm 3.9 * * *$ \\
\hline 4. & Diazepam -2 & $2.8 \pm 0.67 * * *$ & $129 \pm 6.85^{* * *}$ \\
\hline 5. & Ganaxolone 5 + Diazepam 1 & $4.1 \pm 0.72^{* * *}$ & $83 \pm 4.98^{* * *}$ \\
\hline \multicolumn{2}{|r|}{$* * *-p<0.001, * *-p<0.01$ compared to control } \\
\hline
\end{tabular}


Evaluation of Anti - Anxiety, Sedative and Motor Co-Ordination Properties of Ganaxolone in

\begin{tabular}{|c|c|c|c|c|c|}
\hline \multirow[t]{2}{*}{$\begin{array}{l}\text { S. } \\
\text { N.o }\end{array}$} & \multirow[t]{2}{*}{$\begin{array}{l}\text { Group \& Dose } \\
(\mathrm{mg} / \mathrm{kg} \text { bw })\end{array}$} & \multicolumn{2}{|c|}{$\begin{array}{l}\text { Mean Time of fall for the first time in Rota } \\
\text { rod apparatus (sec) }\end{array}$} & \multicolumn{2}{|c|}{$\begin{array}{l}\text { No. of falls in } 5 \text { minutes period } \\
\text { after drug administration }\end{array}$} \\
\hline & & 30 & 60 & 30 & 60 \\
\hline 1. & Control & $247.33 \pm 5.89$ & $250.67 \pm 4.761$ & $0.5 \pm 0.5$ & $0.5 \pm 0.5$ \\
\hline 2. & Ganaxolone - 5 & $239 \pm 7.98$ & $247.5 \pm 8.40$ & $1.7 \pm 0.5$ & $1.3 \pm 0.5$ \\
\hline 3. & Ganaxolone - 10 & $200.33 \pm 11.29 * * *$ & $237.67 \pm 6.25$ & $3.2 \pm 0.8 * * *$ & $1.5 \pm 0.5$ \\
\hline 4. & Diazepam -2 & $36.5 \pm 7.2 * * *$ & $77.83 \pm 13.50 * * *$ & $6.2 \pm 1.2 * * *$ & $4.3 \pm 1.3 * * *$ \\
\hline 5. & $\begin{array}{l}\text { Ganaxolone } 5+ \\
\text { Diazepam } 1\end{array}$ & $89.5 \pm 5.9 * * *$ & $153.7 \pm 8.5^{* * *}$ & $4.5 \pm 1.0 * * *$ & $2.66 \pm 0.81 * * *$ \\
\hline
\end{tabular}

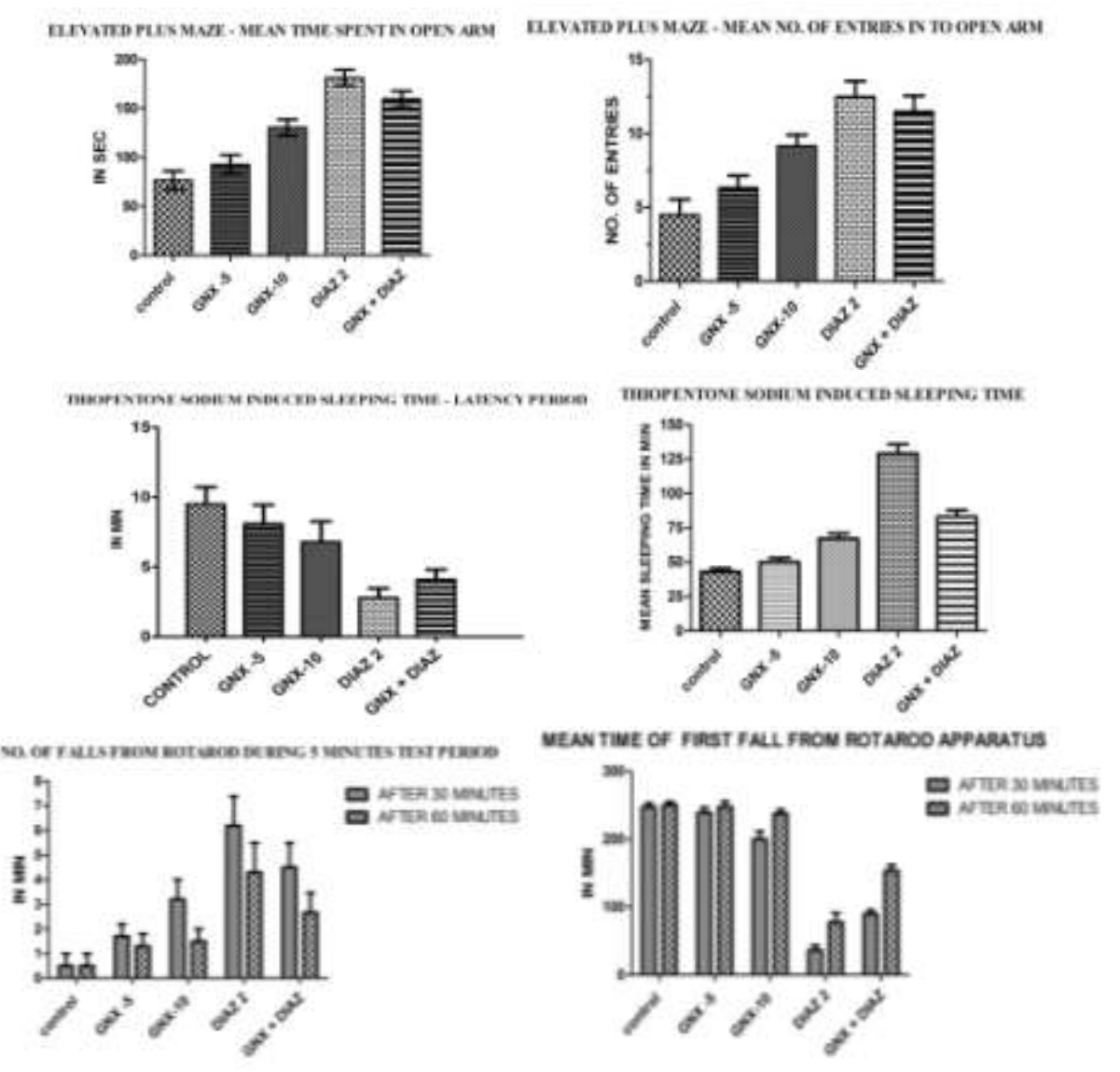

IV. Discussion

Anxiety is a negative emotion that occurs in response to perceived threats that can come from internal or external sources and can be real or imagined [13]. Pharmacological knowledge of ganaxolone would allow us to evaluate the anti-anxiety activity, which could be used to treat anxiety type of disorders. So, the present study was aimed at evaluating the anti - anxiety, sedative and motor co-ordination properties of ganaxolone using elevated plus maze, thiopentone induced sleeping and rotarod model in rats and to compare it with diazepam.

Ganaxolone (GNX) is the synthetic analog of allopregnanolone $(3 \alpha, 5 \alpha-\mathrm{P})$ belonging to a class of compounds referred as neurosteroids that have been termed "epalons" which has been presumed to possess sedative, anxiolytic, and anticonvulsant effects [14].

Elevated plus maze test was used to assess psychomotor performance and emotional aspects of rodents, treatment with ganaxolone $(5$ and $10 \mathrm{mg} / \mathrm{kg}$ ) revealed anxiolytic activity, since the number of open arm entries and time spend in open arm parameters are the most delegate guide for anxiolytic activity [15]. The time spent by the animal in the central platform seem to be related to decision making or risk assessment and the total arm entries is a measure reflecting changes in anxiety [16]. In our study, we demonstrated significant anti-anxiety activity with $10 \mathrm{mg}$ of ganaxolone compared to control group but has less activity when compared to that of diazepam but both were statistically significant. Our findings was supported by, Robert M. Mihalek et al (1999), who proved that, ganaxolone, possess anti-anxiety activity due to potentiating action on $\mathrm{GABA}_{\mathrm{A}}$ receptor mediated inhibitory responses in the brain [17]. 
Thiopentone induced sleeping time was designed to find out the sedative and CNS depressant action of the ganaxolone in two doses of $5 \& 10 \mathrm{mg} / \mathrm{kg}$. In conformity with previous reports $[17,18]$, the results of our study show that the low doses ganaxolone $(5 \mathrm{mg} / \mathrm{kg})$ did not affect thiopentone induced sleeping time where as the dose of $10 \mathrm{mg} / \mathrm{kg}$, significantly increased sleeping time. Wieland et al., (1997) stated that, tolerance has not been observed to the anxiolytic and sedative effects of the synthetic neuroactive steroids ganaxolone, which supports our finding that ganaxolone possess anti anxiety activity \& sedative action [18]. This can be used to overcome one of the adverse effect of diazepam i.e. tolerance.

Rotarod test was first used to screen the neurotoxicity profile of anticonvulsants and later used to calculate motor dysfunction produced by centrally acting drugs to determine possible alterations in the motor coordination ability of the animal, based on the assumption that an animal with normal motor efficiency is able to maintain its equilibrium on a rotating rod [19]. In this test, the difference in the fall of time from the rotating rod is taken as an index of muscle relaxation. Our observations, gave a conclusion that fall of time from rota rod produced by ganaxolone was less significant when compared with diazepam, indicating that ganaxolone is having a weak skeletal muscle relaxant activity. Doodipala S. Reddy et al. (2000) proved that, ganaxolone produced impairment in motor function as assessed with the accelerating rota rod test, in a dose dependent manner but anticonvulsant / antianxiety doses of ganaxolone do not compromise motor performance in rats [20].

The inhibitory action of GABA consists of opening the chloride channels to allow hyperpolarizing of postsynaptic membrane, leading to CNS depression and resulting in sedative and hypnosis activity. Glutamate and GABA are quantitatively the most important excitatory and inhibitory neurotransmitters, respectively, in the mammalian brain. Thus, receptors for these two neurotransmitters are regarded as important targets for psychotropic drugs [5].

Neuroimaging studies have reported reductions in GABA levels and $\mathrm{GABA}_{\mathrm{A}^{-}}$benzodiazepine receptor binding in patients with anxiety disorders [4]. Ganaxolone inhibited binding of the $\mathrm{GABA}_{\mathrm{A}}$ chloride channel ligand and enhanced binding of the benzodiazepine site ligand and the GABA site ligand. Electrophysiological recordings showed that nanomolar concentrations of GNX potentiated GABA-evoked chloride currents in Xenopus oocytes expressing human $\mathrm{GABA}_{\mathrm{A}}$ receptor subunits $\alpha 1, \beta 2, \gamma 2 \mathrm{~L}$, but direct activation of chloride flux occurred only at micromolar concentrations [21,22]. Ganaxolone have unique, noninteracting binding requirements on $\mathrm{GABA}_{\mathrm{A}}$ receptors distinct from those of benzodiazepines and barbiturates [17]. Additionally, the differential response of ganaxolone to diazepam may be due to variability in binding affinity to $\mathrm{GABA}_{\mathrm{A}}$ receptors.

From our study, ganaxolone $10 \mathrm{mg} / \mathrm{kg}$ has showed more meaningful results compared to ganaxolone 5 $\mathrm{mg} / \mathrm{kg}$. We also noted that, ganaxolone had significant synergistic effect with diazepam by potentiating it actions. This result provides a lead for potential benefit of adding ganaxolone to diazepam in the treatment of anxiety, which needs to be explored further. Second, ganaxolone may provide additional benefit in the treatment of anxiety as it has less sedative, muscle relaxant and tolerance compared to diazepam.

\title{
V. Conclusion
}

Overall, Ganaxolone a high-affinity, stereo selective, positive allosteric modulator of $\mathrm{GABA}_{\mathrm{A}}$ receptors has exhibited strong anti-anxiety activity. The present study supports further clinical evaluation of ganaxolone as an anti-anxiety medication alone or as an add on drug with the conventional anti-anxiety drug diazepam to reduce their dose / adverse effects. This study may throw some light for further research to design and develop newer neuroactive steroids with better CNS efficacy.

\section{Acknowledgement} Aldrich.

\author{
The authors are thankful to the staffs and PGs of Narayana Medical College, Nellore and Sigma
}

\section{References}

[1] S. Kasper, Social Phobia: The nature of the disorder, J Affect Disord, 50, 1998, S3-9.

[2] R. C. Kessler, W. T. Chiu, O. Demler, Prevalence, severity, and co-morbidity of 12-month DSM-IV disorders in the National Comorbidity Survey Replication, Arch Gen Psychiatry, 62, 2005, 617-27.

[3] G. Simon, J. Ormel, M. VonKorff and W. Barlow, Health care costs associated with depressive and anxiety disorders in primary care, American Journal of Psychiatry, 152, 1991, 352-357.

[4] Arya Ashwani, Kumar Tarun, Malik Ajay, Hooda Anil, Anxiety disorders: A review, IRJCP, 2(5), $2011,18-23$.

[5] Anxiolytic and hypnotic drugs, in H. P. Rang, Rang and Dale's Pharmacology, 7 ( Spain: Elsevier Churchill, 2012 ) 531-538.

[6] M. Lader, S. Morton. Benzodiazepine problems. Br J Addict, 83, 1991, 823-828.

[7] K. Suresh, S. Anupam, Apigenin: The anxiolytic constituent of Turnera aphrodisiaca, Pharm Biol, 44, 2006, 84-90.

[8] R. L. Macdonald, R. W. Olsen, GABA A receptor channels, Annu. Rev. Neurosci., 17, 1994, 569-602.

[9] V. Nohria, E. Giller, Ganaxolone, Neurotherapeutics, 4, 2007, 102-105.

[10] M. A. Rogawski, D. S. Reddy, Neurosteroids: endogenous modulators of seizure susceptibility, in: Rho JM, Sankar R, Cavazos JE, editors. Epilepsy: scientific foundations of clinical practice, (New York: Marcel Dekker; 2004) 319-355.

[11] Psychotropic and neurotropic activity, in H. Gerhard Vogel (Ed.), Drug discovery and evaluation: Pharmacological assays, 2 (Germany: Springer-Verlag, 2002) 398, 434, 496. 
[12] Doodipala S. Reddy, Michael A. Rogawski, Chronic treatment with the neuroactive steroid Ganaxolone in the Rat induces anticonvulsant tolerance to Diazepam but not to itself, JPET, 295(3), 2000, 1241-1248.

[13] D. K. Moser, The rust of life: Impact of anxiety on cardiac patients, Am J Crit Care, 16, 2007, 361-369.

[14] K.W. Gee, Epalons as anticonvulsants: actions mediated by the GABA-A receptor Complex, Proc West Pharmacol Soc 39, 1996, 55-60.

[15] R. G. Lister, Ethologically-based animal models of anxiety disorders, Pharmacol Ther, 46, 1990, 321-40

[16] S. E. File, Factors controlling measures of anxiety and responses to novelty in the mouse, Behav Brain Res., 125, 2001, 151-157.

[17] Robert M. Mihalek, Pradeep K. Banerjee, Esa R. Korpi, Joseph J. Quinlan, Leonard L. Firestone, et al., Attenuated sensitivity to neuroactive steroids in $\gamma$-aminobutyrate type A receptor delta subunit knockout mice, Proc Natl Acad Sci, 96(22), 1999, 1290512910.

[18] S. Wieland, J. Belluzzi, J. E. Hawkinson, D. Hogenkamp, R. Upasani, L. Stein, et al., Anxiolytic and anticonvulsant activity of a synthetic neuroactive steroid Co 3- 0593. Psychopharmacology, 134, 1997, 46-54.

[19] N. Tomomi, T. Tomoko, T. Kenshi, K. Chiaki, Evaluation of anxiolytic-like effects of some short-acting benzodiazepine hypnotics in mice, J Pharmacol Sci, 107, 2008, 349-54.

[20] Doodipala S. Reddy, Michael A. Rogawski, Enhanced Anticonvulsant Activity of Ganaxolone after Neurosteroid Withdrawal in a Rat Model of Catamenial Epilepsy, JPET, 294(3), 2000, 909-15.

[21] S. Liptakova, L. Velisek, J. Veliskova, S. L. Moshe, Effect of Ganaxolone on flurothyl seizures in developing rats, Epilepsia, 41, 2000, 788-93.

[22] M. Kaminski, M. R. Livingood, M. A. Rogawski, Allopregnanolone analogs that positively modulate GABA receptors protect against partial seizures induced by 6-Hz electrical stimulation in mice, Epilepsia, 45, 2004, 864-67. 